\title{
Normatização dos Equipamentos e Técnicas para a Realização de Exames de Eletrocardiografia e Eletrocardiografia de Alta Resolução
}

\author{
Coordenador \\ Jorge Ilha Guimarães \\ Editores \\ Paulo J. Moffa \\ Augusto H. Uchida \\ Membro \\ Paulo Benchimol Barbosa
}

Uma grande variedade de dispositivos está disponível no mercado para a realização de eletrocardiograma (ECG) e de eletrocardiograma de alta resolução (ECGAR). É essencial que tais dispositivos sejam validados e qualificados em relação à sua exatidão, para que possamos reconhecer e entender melhor as limitações técnicas de cada método, evitando erros diagnósticos com conseqüências danosas ao paciente. Nosso propósito foi o de contribuir com uma base científica que fundamente a acurácia dos métodos em questão, sem, no entanto, avaliar individualmente aparelhos específicos. Essa tarefa de validação individual será realizada em uma segunda fase, quando somente os equipamentos que alcancem o nível $\mathrm{A}$ ou $\mathrm{B}$ de concordância, mostrados na tabela 1 , serão recomendados para a utilização clínica. Os aparelhos receberão a classificação APROVADO ou REPROVADO, dependendo da diferença média entre o padrão e um dado dispositivo-teste. Nessa fase, serão considerados também os estudos clínicos e laboratoriais de validação, além dos critérios da Associação para o Progresso da Instrumentação Médica (AAMI). Será ainda levada em conta a circunstância da avaliação. Assim, por exemplo, dispositivos destinados a grávidas, crianças, idosos deverão ser validados nessas populações específicas.

Tivemos também uma preocupação com a terminologia utilizada na emissão de laudos, de modo a garantir uma uniformidade terminológica. Nossas recomendações são baseadas em evidências, embora não utilizemos a classificação dos níveis de evidência do ACC/AHA (Classes I, IIa, IIb, III) devido ao pequeno número de estudos sobre o tema. Quando não foram encontradas evidências, foi utilizada a opinião de um especialista. Indicações ou contraindicações do exame não foram incluídas no escopo deste documento.

Não houve a pretensão de uma avaliação perfeita das especificações técnicas, enfocando-se apenas os aspectos clinicamente relevantes da questão, uma vez que a análise de um equipamento médico é complexa, multidimensional e multiprofissional.

Esta normatização foi realizada e revista por autores que são especialistas na área de Eletrocardiologia, sendo desenvolvida de forma imparcial a fim de evitar qualquer tipo de relacionamento que poderia ser percebido como real ou potencial conflito de interesse.

\section{Introdução}

Um aparelho de eletrocardiografia é um instrumento ou sistema de aquisição e processamento da atividade elétrica cardíaca. Os sinais elétricos do coração são adquiridos através de eletrodos aplicados na superfície do tórax. Tais sinais são filtrados e processados por meio de sistemas computadorizados, gerando expressões gráficas e numéricas, que são interpretadas pelo médico. Portanto, a avaliação de um aparelho de eletrocardiografia deve contemplar todas as partes necessárias para obter, processar e demonstrar a atividade elétrica cardíaca. A demonstração do resultado poderá ser visualizada em tela, devendo permitir a sua impressão em papel, além de possibilitar a edição do dado processado pelo médico/operador.

Os aparelhos devem ser avaliados quanto à eficácia e segurança, o que inclui a análise das características de acurácia e reprodutibilidade, além de quaisquer limitações para a documentação da atividade elétrica cardíaca.

Com respeito à eficácia, o foco primário da análise recaiu sobre o risco potencial de erro diagnóstico relacionado a uma falsa medida.

A análise da acurácia compreendeu a medida em amplitude e tempo, influência de ruídos, linearidade, calibração, além de controles e recursos que minimizam potenciais erros por parte do operador do aparelho. Considerações sobre segurança contemplam a análise de cabos-eletrodos, fio terra e sistemas de proteção dos circuitos elétricos do aparelho.

Âmbito, restrições e limitações - Foram considerados os tipos de equipamentos com impressão térmica acoplada e aqueles baseados em PC, ou seja, com sistemas acoplados a microcomputadores. Não foi objeto desta normatização a análise específica dos eletrodos e da técnica dos sistemas de impressão utilizados na realização dos exames. Não foram consideradas as especificações dos aparelhos e técnicas de exame da vetorcardiografia, eletrocardiografia dinâmica (Holter), eletrocardiografia de esforço, eletrocardiografia de monitorização transtelefônica, mapeamento eletrocardiográfico de superfície, eletrocardiografia epicárdica, intra- 


\begin{tabular}{|cl|}
\hline Tabela I. Graus de concordância (dispositivo padrão e dispositivo teste) \\
\hline A & Melhor concordância \\
B & Concordância excelente \\
C & Concordância deficiente \\
D & Sem concordância \\
\hline
\end{tabular}

cardíaca, esofágica e fetal. Não foi contemplada também a avaliação de monitores eletrocardiográficos.

\section{Eletrocardiograma de repouso}

O eletrocardiograma (ECG) de repouso recebe várias denominações: ECG basal, ECG de superfície, ECG convencional ou, simplesmente, eletrocardiograma. Trata-se de exame não-invasivo, de baixo custo e de realização rápida e fácil. Apesar de inúmeras inovações tecnológicas que vêm sendo incorporadas na prática da medicina cardiovascular, o ECG permanece como o recurso diagnóstico mais amplamente utilizado na avaliação dos distúrbios do ritmo cardíaco. Esse exame, através do registro da atividade elétrica do coração, pode também prover informações sobre o diagnóstico de problemas estruturais cardíacos, isquemia miocárdica, distúrbios eletrofisiológicos do miocárdio, pericardiopatias, posicionamento do coração, estimulação cardíaca artificial, alterações metabólicas e eletrolíticas sistêmicas, além de documentação de influências autonômicas e farmacológicas (tóxicas ou terapêuticas).

$\mathrm{O}$ traçado eletrocardiográfico tem uma representação gráfica, que possibilita distinguir diferentes configurações morfológicas e do ritmo cardíaco. Será utilizada a seguinte terminologia padrão para os laudos de eletrocardiograma:

Ondas: deflexões P, QRS (complexo), T, Ta (T atrial), $\mathrm{U}$, fe F; além das ondas delta, epsílon, J de Orborne e, mais recentemente, a onda em cela (V1 e V2) - sinal de síndrome de Brugada.

Intervalos ou espaços: $\mathrm{P}-\mathrm{R}$ (PRi.); QT. QTc-QT corrigido pela freqüência, sexo e idade; deflexão intrinsecóide ou tempo de ativação ventricular.

Segmento: segmento PR (PRs) ou segmento. O ponto J demarca o final do complexo QRS e o início do segmento ST.

Espículas: marcações da estimulação cardíaca artificial ou da cardiomioplastia.

Entalhes: pequenas demarcações que podem detalhar as ondas ou deflexões do ECG.

Artefatos musculares: vibrações, correspondentes a pequenos sinais de alta freqüência (tremores musculares).

Assistolia: ausência de atividade elétrica ventricular.

Fibrilação: impossibilidade de identificação de ondas que definam morfologicamente as atividades atrial (fibrilação atrial) ou ventricular (fibrilação ventricular).

Eletrocardiograma morfologicamente normal: ECG normal, ECG dentro dos limites da normalidade, traçado compatível com a normalidade, ECG normal para a idade e biótipo.

Problemas técnicos de registro: troca de eletrodos, artefatos.

Distúrbios da condução intraventricular: pré-excita- ção ventricular, síndrome de Wolff-Parkinson-White, WPW (localização provável- lateral esquerda, lateral direita, póstero-septal esquerda, póstero-septal direita, médio septal, ântero-septal), atraso final da condução, distúrbio da condução pelo ramo direito, bloqueio completo do ramo direito, bloqueio divisional ântero-superior, bloqueio divisional póstero-inferior, bloqueio divisional ântero-medial, bloqueio completo do ramo esquerdo, distúrbio da condução intraventricular (inespecífico).

Sobrecargas: sobrecarga atrial direita, sobrecarga atrial esquerda, sobrecarga ventricular direita, sobrecarga ventricular esquerda, sobrecarga de câmaras direitas, sobrecarga de câmaras esquerdas, possível sobrecarga atrial, possível sobrecarga ventricular.

Posicionamento, eixo elétrico e voltagem: eixo indeterminado, alternância elétrica, baixa voltagem, dextrocardia.

Alterações da repolarização ventricular e infarto do miocárdio: repolarização precoce (normal variante), onda $\mathrm{T}$ juvenil (variante normal), onda T cerebral, alterações inespecíficas da repolarização ventricular, alterações da repolarização ventricular, alterações difusas da repolarização ventricular, alterações difusas discretas da repolarização ventricular, QT longo, área eletricamente inativa (localização), infarto do miocárdio (localização), bloqueio de lesão.

Condições clínicas: pericardite, sugestivo de hipocalemia, sugestivo de hipercalemia, sugestivo de hipocalcemia, sugestivo de hipercalcemia.

Distúrbios do ritmo e da condução $A V$ : ritmo sinusal bradicárdico, ritmo sinusal taquicárdico, ritmo ectópico atrial, arritmia sinusal respiratória, arritmia sinusal ventriculo-fásica, marcapasso atrial mutável, extra-sístole supraventricular, extra-sístole atrial, extra-sístole ventricular, bigeminismo atrial, bigeminismo ventricular, PR curto, bloqueio atrioventricular de $1^{\circ}$ grau, bloqueio atrioventricular de $2^{\circ}$ grau, bloqueio atrioventricular de $2^{\circ}$ grau Mobitz 1 (fenômeno de Wenckebach), bloqueio atrioventricular de $2^{\circ}$ grau Mobitz 2, bloqueio atrioventricular de $2^{\circ}$ grau fixo: 2:1, 3:1, 4:1; bloqueio atrioventricular de $2^{\circ}$ grau avançado, bloqueio atrioventricular de $3^{\circ}$ grau ou bloqueio atrioventricular total, bloqueio sinoatrial, bloqueio sinoatrial de $2^{\circ}$ grau Mobitz 1 , bloqueio sinoatrial de $2^{\circ}$ grau Mobitz 2, pausa sinusal, síndrome bradicardia-taquicardia, taquicardia ventricular nãosustentada, ritmo idioventricular, taquicardia supraventricular sustentada, taquicardia paroxística supraventricular, taquicardia juncional, fibrilação atrial, flutter atrial, taquicardia atrial, taquicardia atrial com condução $\mathrm{AV}$ variável, taquicardia atrial multifocal, taquicardia de reentrada nodal, taquicardia atrioventricular ou reciprocicante, taquicardia atrioventricular forma incessante de Coumel, taquicardia ventricular sustentada, taquicardia ventricular monomórfica, taquicardia ventricular polimórfica, torsade des pointes, taquicardia ventricular bidirecional, taquicardia ventricular de via de saída do ventrículo direito, taquicardia ventricular fascicular.

Fenômenos: aberrância de condução, fenômeno de Wenckebach, fenômeno de Ashman, alternância elétrica, alternância de onda $T$, pseudobloqueio atrioventricular, dissociação atrioventricular, batimentos de fusão, captura, parassistolia. 


\section{Eletrocardiografia de marcapasso}

Terminologia para laudo: $\mathrm{FE}$ = freqüência de estimulação, blanking ventricular, $\mathrm{IAV}=$ intervalo atrioventricular, IAV diferencial, IAV dinâmico, Intervalo de escape (pulso) do marcapasso, $\mathrm{ppm}=$ pulsos por minuto, FEmag $=$ freqüência de estimulação magnética, FEmáx = freqüência de estimulação máxima, FEmín = freqüência de estimulação mínima, $\mathrm{ME}=$ modo de estimulação, $\mathrm{MPW}=$ mecanismo de pseudo-wenckebach, $\mathrm{PEPVARP}=$ período refratário atrial pós-ventricular pós-extra-sistólico, $\mathrm{PRA}=$ período refratário atrial, $\mathrm{PRV}=$ período refratário ventricular, $\mathrm{PRAT}=$ período refratário atrial total, PVARP = período refratário pósventricular, $\mathrm{RS}=$ rate smoothing, runaway, relação $\mathrm{AV}=$ relação atrioventricular, teste de limiar de estimulação, teste de margem de segurança.

Frases para laudo: marcapasso artificial normofuncionante (modo de operação-AOO, AAIC, AAIR, VOO, VVIC, VVIR, VDD,DOO,DVIC,DVIR,DDDC,DDIC,DDDR), automatic mode switching, perda de captura, oversensing atrial, undersensing atrial, oversensing ventricular, oversensing de $\mathrm{T}$, undersensing ventricular, pseudofusão atrial, pseudofusão ventricular, taquicardia mediada pelo marcapasso, taquicardia conduzida pelo marcapasso, taquicardia induzida pelo marcapasso, safety pace ativado, Crosstalk, fusão atrial, fusão ventricular, função vario ativada, inibição da estimulação por miopotenciais, histerese programada para $\mathrm{FC}=x \mathrm{bpm}$, estimulação multissítio.

Derivações eletrocardiográficas. Todo o traçado eletrocardiográfico deverá conter, independente de seu formato, o registro das derivações D1, D2, D3, aVR, aVL, aVF, $\mathrm{V} 1, \mathrm{~V} 2, \mathrm{~V} 3, \mathrm{~V} 4, \mathrm{~V} 5$ e V6. Um período de registro do ritmo é obrigatório, utilizando-se, ao menos, uma derivação D2. Derivações especiais podem ser registradas, conforme contexto clínico. Serão consideradas derivações especiais: V3r, V4r, V7, V8, derivações de Lewis, de Golub, epicárdica, esofágica e eletrolítica.

Tipo de registro eletrocardiográfico. A apresentação do ECG deverá ser realizada, preferencialmente, no formato com orientação vertical, com registro de, no mínimo, três canais simultâneos. É desejável que se associe ao registro uma derivação de ritmo D2.

Interpretação do ECG. A interpretação e laudo do ECG deverão ser realizados por médico competente, que, obrigatoriamente, irá considerar a idade, sexo e contexto clínico na sua análise. A interpretação computadorizada do ECG é ainda considerada inapropriada.

\section{O eletrocardiograma de alta resolução(ECGAR)}

OECGAR tambémé conhecido como eletrocardiograma promediado (do inglês Signal Averaged Electrocardiography) ou eletrocardiograma de alta freqüência. Constitui método não-invasivo e de fácil aplicação clínica, com finali- dades principais de melhor avaliação da onda $\mathrm{P}$, atividade do sistema de His-Purkinje e a detecção de potenciais tardios (PT). É oportuno lembrar que tais potenciais documentados pelo ECGAR não correspondem aos pós-potenciais precoces ou tardios verificados numa curva de potencial de ação monofásico.

Sua principal função é reduzir resíduos de sinais amostrados, conservando os sinais de alta freqüência e baixa amplitude, que denotam uma fragmentação de atividade elétrica ventricular e que são identificados na parte terminal do complexo QRS e no início do segmento ST, mas não são evidentes no eletrocardiograma convencional com ganho nor$\mathrm{mal}(10 \mathrm{~mm}$ por $\mathrm{mV}$ e velocidade do papel de $25 \mathrm{~mm} / \mathrm{s})$. No ECGAR, quando o sinal elétrico é processado mais profundamente, ou seja, utilizando amplificação de alto ganho de até 100 vezes, ou 1 microvolt por milímetro, e grande aumento da velocidade de aquisição do sinal, o PT pode ser claramente identificado na parte final do QRS. Por outro lado, verifica-se PT ausente quando a atividade elétrica não se apresenta fragmentada. PT ou despolarização retardada corresponde à verdadeira atividade elétrica ventricular arritmogênica latente.

Um processo da promediação do sinal elétrico cardíaco é realizado para a detecção de pequenos sinais elétricos constituintes do complexo QRS, eliminando-se os ruídos contaminantes provenientes do eletrocardiograma de superfície. As fontes de ruídos incluem os miopotenciais da superfície corpórea, a interface pele-eletrodos, amplificadores e correntes de 60 ciclos e seus sinais harmônicos.

O tipo mais comum do processamento inclui a promediação no domínio do tempo, na qual múltiplos complexos QRS amplificados são somados no tempo. Para que essa técnica torne-se válida e clinicamente aplicável, algumas recomendações devem ser obedecidas: 1) os sinais devem ocorrer de forma homogênea e repetitiva; 2 ) devem respeitar um ponto fiducial comum de referência; 3 ) o ruído deve ser aleatório, com distribuição gaussiana; 4) a redução do ruído deve ser proporcional à raiz quadrada do número de complexos QRS promediados (por exemplo: 100 complexos obtidos resultam numa redução de ruído por um fator de 10).

As derivações usadas para obtenção desses procedimentos são ortogonais (aproximadas), constituindo os eixos X, YeZ.

Após a promediação temporal, os complexos são filtrados para que se eliminem as freqüências baixas. Os filtros utilizados são do tipo bidirecional, para evitar os artefatos ou vibrações. As interferências de artefatos de baixa freqüência caem abruptamente com a utilização de filtragem de $10-20 \mathrm{~Hz}$, diminuindo, gradualmente, com $20-50 \mathrm{~Hz}$ e tornando-se estáveis em níveis de filtragem de $50-100 \mathrm{~Hz}$.

Deverão ser utilizadas as três derivações ortogonais bipolares X, Y, Z, com amplificação dos sinais elétricos de 200 a 300 batimentos promediados (média coerente) e submetidos a filtros digitais bidirecionais de faixa ampla, com a finalidade de evitar vibrações (filttro passa alta acima de $40 \mathrm{~Hz}$ e passa baixa abaixo de $250 \mathrm{~Hz}$ ). Os sinais filtrados das três derivações são convertidos em um vetor de magnitude, que 
corresponde à raiz quadrada das somas da voltagem das três derivações ao quadrado $\left(\mathrm{M}=\sqrt{\mathrm{x}^{2}+\mathrm{y}^{2}+\mathrm{z}^{2}}\right)$ e que é conhecido como $\mathrm{QRS}$ filtrado ou depurado. Três parâmetros deverão ser detectados no complexo QRS depurado, seguindo a seguinte ordem de importância: A) duração do complexo QRS; B) voltagem espacial da raiz quadrada dos últimos 40ms; C) duração do sinal de baixa amplitude (abaixo de $40 \mathrm{mV}$ ), na porção terminal do complexo QRS.

Critérios de positividade (filtros $40 \mathrm{~Hz}$ ): admite-se potencial tardio (PT) presente, se dois dos seguintes critérios forem encontrados: 1) duração do QRS filtrado maior do que $114 \mathrm{~ms}$; 2) voltagem do sinal inferior a 20 microV nos últimos 40ms do QRS filtrado; 3) nos últimos $38 \mathrm{~ms}$ do QRS filtrado, a magnitude dos sinais deve permanecer abaixo da linha de 40microV.

O ECGAR tem uma representação gráfica,através da qual se podem distinguir diferentes configurações morfológicas, sem possibilidade de análise do ritmo cardíaco. Considera-se a seguinte terminologia padrão para os laudos de ECGAR:

- quando se atingem dois ou mais critérios de positividade: PT presente;

- quando não se atingem dois ou mais critérios de positividade: PT ausente (dos três critérios utilizados, o de maior valor diagnóstico é a duração do QRS filtrado);

- quando há contaminação excessiva do sinal promediado: ECGAR inconclusivo;

- quando os valores de dois ou mais critérios estão no limite superior da normalidade: ECGAR limitrofe (jamais desprezar o nível de ruído gerador do referido exame).

OECGAR nos bloqueios de ramo - A limitação do uso do ECGAR no domínio do tempo nos portadores de bloqueio de ramo relaciona-se à não uniformidade de critérios para reconhecimento do PT. Não existe um consenso estabelecido quanto à utilização do número de parâmetros anormais e ao tipo de filtro utilizado $(25,40 \mathrm{ou} 80 \mathrm{~Hz})$ para que um teste seja considerado positivo na presença de bloqueio completo de ramo (direito ou esquerdo).

Por outro lado, na presença de bloqueio de ramo, o substrato arritmogênico de uma eventual TV, assim como o comportamento dos bloqueios de ramo apresentam efeitos semelhantes no ECGAR. Esse fato tem levado alguns pesquisadores a estabelecerem critérios mais rígidos para o correto reconhecimento do PT em casos de bloqueio de ramo. Analisando-se pacientes com duração de QRS basal (não filtrado) $\geq 120 \mathrm{~ms}$ e utilizando filtros de $25 \mathrm{a} 250 \mathrm{~Hz}$, considerou-se a presença de PT quando todos os três parâmetros utilizados no ECGAR estivessem presentes, além de: a) duração do complexo QRS filtrado $\geq 120 \mathrm{~ms}$; b) duração total dos sinais de baixa amplitude inferior a $40 \mu \mathrm{V}, \geq 40 \mathrm{~ms}$ e voltagem média dos 40 ms terminais do complexo QRS filtrado $\leq 15 \mu \mathrm{V}$.

O ECGAR no domínio da freqüência - A análise do ECGAR no domínio da freqüência originou-se na possibilidade de superar as limitações do ECGAR no domínio do tempo e que levavam, por exemplo, à necessidade de filtros específicos. A análise do domínio da freqüência do segmento ST baseia-se na hipótese de que os sinais fragmentados em áreas arritmogênicas são de freqüências diferentes dos sinais normais de baixa freqüência do segmento ST de áreas do miocárdio normal.

Essa metodologia foi abandonada tendo em vista a delineação subjetiva do intervalo analisável e a variação do comprimento do segmento ST de um paciente para outro. Assim, uma alteração de $3 \mathrm{~ms}$ na identificação do QRS é suficiente para provocar alterações significativas nos resultados da análise no domínio da freqüência. Outra causa para seu abandono foi o desenvolvimento de novas técnicas no domínio da freqüência, como a análise da turbulência espectral ou espectrocardiografia.

\section{Espectrocardiografia}

Essa técnica difere das demais, pois não procura identificar ou medir um PT, nem pressupõe qualquer correlação de regiões cardíacas arritmogênicas com determinadas freqüências ou situações peculiares no complexo QRS. As observações, medidas e cálculos são realizados após a aquisição do sinal do complexo QRS por inteiro e não em qualquer porção arbitrariamente identificada por características de tempo, freqüência ou amplitude. Enfim, os critérios não são dependentes de fatores subjetivos.

A espectrocardiografia detecta flutuações anormais na composição de freqüências da onda de despolarização, que se propagam através e em torno de áreas lesadas do miocárdio e que formam o substrato para a reentrada, seja qual for a sua localização no complexo QRS. Resumindo, apesar de aparente complexidade, o método analisa o grau de alterações transitórias no conteúdo de freqüências durante a despolarização de todo o ventrículo (turbulência espectral), considerada um indicador da presença de substrato arritmogênico.

O método utiliza as derivações $\mathrm{X}, \mathrm{Y}, \mathrm{Z}$, processadas separadamente, fornecendo gráficos tridimensionais representativos do conteúdo de freqüências, que constituem os dados de freqüência espectral, denominados "espectrocardiogramas". Os dados de cada derivação são submetidos a vários cálculos estatísticos, através de informatização (incluindo a transformada rápida de Fourier), que, por sua vez, fornecem índices numéricos para compor uma escala ascendente de turbulência espectral. Como se trata de técnica de aquisição recente, é obvia a necessidade de um maior número de estudos para sua fundamentação.

\section{ECGAR de onda $P$}

OECGAR de onda P, no domínio do tempo, está sendo utilizado para avaliações de potenciais tardios atriais, que são sinais elétricos de baixa amplitude e alta freqüência na porção terminal da ativação atrial, indicativos de risco para o desenvolvimento de fibrilação atrial. Os parâmetros de maior sensibilidade e especificidade são duração da onda $P$ filtrada $\geq 130 \mathrm{~ms}$ e duração dos sinais da onda $\mathrm{P}$ abaixo de 03 microV $\geq 10,61 \mathrm{~ms}$. 


\section{Equipamentos de eletrocardiografia (ECG E ECGAR)}

\section{Pré-requisitos}

Para a utilização na prática clínica, os aparelhos de eletrocardiografia devem estar aprovados por órgãos normativos que qualificam não só o produto, como o processo de fabricação industrial. Nos EUA, fica eleita a aprovação pelo FDA e, no Brasil, a aprovação pelo INMETRO.

\section{Requisitos}

Serão considerados requisitos fundamentais de cada aparelho: 1) etiqueta de especificação técnica, com as informações obrigatórias sobre fabricante, marca registrada, modelo do aparelho, número de série, faixa de freqüência, amperagem, corrente de voltagem aceita; 2) painel de controle e apêndices, tais como os cabos-eletrodos e seus locais de conexão, devidamente identificados. Em casos de simbolização dos controles e cabos, a legenda deverá estar aplicada em local de fácil visualização. Os cabos-eletrodos deverão ter impressa, em seus extremos, a polarização, positiva ou negativa. É desejável que o painel contenha um esquema didático de posicionamento dos cabos-eletrodos no tórax do paciente; 3) manual do operador com instruções adequadas para a instalação e manutenção, além de orientações para a obtenção de um resultado acurado e seguro. O manual deverá informar também sobre todas as funções e aplicações do aparelho, significado de quaisquer sinais e símbolos impressos, especificações técnicas, detalhamento do modelo e cuidados especiais, como relativos à exposição a material inflamável, uso sob interferência eletromagnética e sob situações de sobrecarga elétrica.

\section{III) Condições de operação}

O aparelho de eletrocardiografia deve permitir o registro da atividade elétrica do coração em pacientes dos mais diversos biótipos e com quaisquer conformações da caixa torácica.

Aspectos fundamentais que devem ser avaliados: 1) estabilidade da linha de base, movimentação do paciente; 2) filtro muscular, filtro de freqüência; 3 ) registros em pacientes críticos; 4) influência de ruído e de campo eletromagnético aceitável; 5) influência do posicionamento dos eletrodos; 6) proteção contra descarga de desfibrilador ou sobrecarga de corrente elétrica; 7) portabilidade; 8) outros recursos: envio (transmissão de arquivos) e sistema de laudo automático.

\section{IV) Resultados}

As informações resultantes da aquisição e processamento do sinal deverão ser demonstradas com conteúdo gráfico e numérico. Quanto à obtenção do ECG, o conteúdo gráfico deverá contemplar o registro das 12 derivações clás- sicas (D1, D2, D3, aVR, aVL, aVF, V1 a V6), e o conteúdo numérico será a determinação da freqüência cardíaca em batimentos por minuto. Quanto à obtenção do ECGAR, o conteúdo gráfico deverá contemplar gráficos dos complexos QRS nas derivações X, Y eZ, os complexos QRS promediados e a magnitude vetorial representativa da integral de $\mathrm{X}, \mathrm{Y}$ e Z, aplicando o teorema de Pitágoras. O conteúdo numérico deverá conter dados de calibração (freqüência, velocidade e amplitude), duração do QRS filtrado, valor do RMS em microV (Root Mean Square), número de ciclos cardíacos promediados (mínimo 200 para validar o exame), duração dos sinais de baixa amplitude abaixo dos 40 microV. O nível de ruído aceitávelé, no máximo, de 0,3 microV. Também devem constar do resultado do ECG e do ECGAR dados de identificação do paciente, sexo, idade, data e hora do exame.

\section{Eletrocardiógrafos}

As correntes elétricas geradas pelo coração são de pequena amplitude e incapazes de movimentar, com a devida velocidade e intensidade, os galvanômetros comuns. Torna-se necessário, portanto, recorrer a artifícios que amplifiquem essas correntes. De uma maneira geral, os eletrocardiógrafos têm amplificação óptica ou eletrônica.

Eletrocardiógrafos de amplificação óptica - Trata-se do galvanômetro de corda, que foi aperfeiçoado por Einthoven em 1903 e constitui o ponto real de partida para a eletrocardiografia atual. Um dos inconvenientes desse aparelho é a relativa fragilidade de sua corda de quartzo. Outros dois inconvenientes são o traçado obtido em papel fotográfico, que exige revelação e secagem, retardando a leitura do traçado e a resistência da pele do paciente, que é um fator importante nesse tipo de aparelho. A pele precisa ser cuidadosamente preparada antes do registro do traçado, reajustando-se a sensibilidade do aparelho em cada derivação.

Eletrocardiógrafos de amplificação eletrônica - Os tipos fundamentais deste grupo são: a) Galvanômetro de espelho: Este aparelho tem uma amplificação mista: uma eletrônica predominante e outra óptica, de menor grau. Geralmente, esses modelos são alimentados por baterias, sendo próprios para locais sem luz elétrica. Apesar da desvantagem da revelação do filme, a sua elevada resposta dinâmica torna-os ideais para experimentação; $b$ ) Galvanômetro de bobina: Um estilete preso à bobina permite a inscrição direta do ECG, seja por meio de tinta ou de um estilete aquecido. O peso deste sistema exige uma amplificação maior e o uso de amplificadores mais perfeitos. Sua inércia limita a resposta às freqüências mais elevadas. Embora excelente para o uso clínico, a limitação apontada torna esse modelo inapropriado para a experimentação em pequenos animais ou para o registro de traçados com eletrodos diretamente em contato com o coração. A vantagem dos aparelhos que empregam a inscrição por tinta é que, em lugar do filme, usam papel comum, de baixo custo e fácil obtenção, com as coordenadas já impressas,. O emprego de papéis termossensí- 
veis e de estiletes aquecidos, associados a um mecanismo de correção tangencial, permite o registro de um traçado em tudo semelhante ao habitualmente obtido com sistemas fotográficos, razão pela qual esses aparelhos têm grande aceitação na prática diária.

\section{Técnicas de exame}

Aspectos técnicos do registro - Um grande número de fatores técnicos pode alterar a qualidade e acurácia do registro, alguns relacionados ao paciente, outros ao operador e ao equipamento.

Técnica de registro do ECG de repouso - Para a obtenção de um traçado eletrocardiográfico em condições técnicas satisfatórias, impõem-se as seguintes regras básicas:

1. Posicionamento: o decúbito dorsal horizontal deverá ser o posicionamento padrão para a obtenção do registro eletrocardiográfico. Registros obtidos nas posições ortostática e sentada não deverão ser adotados como traçados de referência para análise do eixo elétrico do QRS (SAQRS) e da repolarização ventricular.

2. Ambiente: o paciente não deverá estar em contato com partes de metal da maca ou divã; permanecendo imóvel e em posição confortável, a fim de evitar tremores musculares. O local deve ser, preferencialmente, afastado de aparelhos de ondas curtas, fios de alta tensão, motores e outros aparelhos elétricos, pois esses causam interferências no traçado. A tomada de corrente elétrica para ligar o aparelho deve permitir um contato com entrada para fio terra.

3. Preparo da pele: é conveniente limpar, com álcool, benzina ou éter, o local da aplicação dos eletrodos, especialmente quando se emprega aparelho de amplificação óptica. Após a limpeza da pele, recomenda-se a aplicação de, aproximadamente, $1 \mathrm{~cm}$ de pasta apropriada. Aplicada a pasta, convém friccionar a pele do local, numa área não superior a $1 \mathrm{~cm}^{2}$.

\section{Registro eletrocardiográfico}

\section{Coordenadas do papel de registro}

No traçado eletrocardiográfico, o tempo é registrado por linhas verticais, separadas umas da outras por um intervalo constante, correspondente a um valor de tempo préfixado. A voltagem, por sua vez, é relacionada com linhas horizontais, também separadas entre si por um intervalo fixo, que corresponde a um determinado valor de voltagem. Dessa maneira, é construído um sistema de abscissas e ordenadas, formando um quadriculado sobre o papel.

Nos papéis comuns, de registro direto, o quadriculado obedece à seguinte disposição: cada traço (tanto os verticais como os horizontais) dista do outro $1 \mathrm{~mm}$, formando assim, pequenos quadrados de $1 \mathrm{~mm}$ de lado. Entre cada grupo de 5 quadradinhos, existe um traço mais grosso, que delimita quadrados maiores, de $0,5 \mathrm{~cm}$ de lado.
Na prática diária, usa-se imprimir uma velocidade padrão ao deslocamento do papel. Essa velocidade é de $25 \mathrm{~mm} / \mathrm{s}$. Existem aparelhos que permitem utilizar velocidades maiores, tais como 50 ou até $100 \mathrm{~mm} / \mathrm{s}$, que são usadas para estudos de certos detalhes da inscrição.

Com a velocidade de $25 \mathrm{~mm} / \mathrm{s}$, cada milímetro passa a valer $0,04 \mathrm{~s}$. Dessa forma, um grupo de $5 \mathrm{~mm}$ representa $0,20 \mathrm{~s}$.

\section{Técnica de registro do ECG de repouso em crianças}

Para a obtenção de um exame em condições técnicas satisfatórias, impõem-se as seguintes regras básicas:

- Usar eletrodo com cola adesiva apropriada, descartável e de tamanho adequado, com conformação específica para neonatos ou para uso pediátrico.

- Posicionar rigorosamente os eletrodos precordiais.

- Evitar uso excessivo de pasta de contato. A pasta pode facilmente se estender pelo precórdio, gerando um campo elétrico homogêneo, o que criará uma morfologia semelhante para todos os complexos QRS de V1 a V6.

- A critério médico, utilizar sedação, quando não for possível obter traçados com qualidade técnica satisfatória.

\section{Técnica de registro do ECGAR}

Para a obtenção de um exame em condições técnicas satisfatórias, impõem-se as seguintes regras básicas:

1) Posicionamento: o decúbito dorsal horizontal deverá ser o posicionamento padrão para a realização do exame.

2) Ambiente: o paciente não deverá estar em contato com partes de metal da maca ou divã; permanecendo imóvel e em posição confortável, a fim de evitar tremores musculares. O local deve ser, preferencialmente, afastado de aparelhos de ondas curtas, fios de alta tensão, motores e outros aparelhos elétricos que geram campos eletromagnéticos, pois esses causam interferências no aparelho que inviabilizam o exame. A tomada de corrente elétrica para ligar o aparelho deve permitir um contato com entrada para fio terra.

3) Preparo da pele: é conveniente limpar-se com álcool o local da aplicação dos eletrodos. Em caso de interferências, fica recomendada a utilização de lixa de pele.

\section{Fatores que podem prejudicar o exame (ECG e ECGAR)}

I. Fatores relacionados ao paciente: 1) movimentação que gera artefatos; 2) conformação do tórax.

II. Fatores relacionados ao operador: 1) posicionamento dos eletrodos; 2) uso excessivo de pasta de contato

\section{Fatores relacionados ao equipamento}

1) sistema de calibração - antes de iniciar o registro do ECG, deve-se padronizar o aparelho com referência à voltagem. Para isso, existe um dispositivo elétrico que introduz 
uma corrente perfeitamente conhecida $(1 \mathrm{mV})$ no circuito. Para o registro do ECG, é obrigatório calibrar o aparelho de modo que a $1 \mathrm{mV}$ corresponda um deslocamento de $1 \mathrm{~cm}$ da linha base. Dessa maneira, quando é introduzido o padrão elétrico no sistema, obtém-se um deslocamento de 10 linhas horizontais $(10 \mathrm{~mm}=1 \mathrm{~cm})$, ou seja, 2 linhas grossas. Cada 1 $\mathrm{mm}$ passa a valer, portanto, $0,1 \mathrm{mV}$ no sistema das ordenadas. Quando as deflexões em uma determinada derivação são muito amplas e tendem a ultrapassar os limites do papel, usa-se diminuir a referência padrão, fazendo com que cada 1 $\mathrm{mV}$ corresponda a $5 \mathrm{~mm}$, isto é, metade do padrão anterior. Como, na prática, a correlação $1 \mathrm{mV} 10 \mathrm{~mm}$ é a mais usada, diz-se que é a referência normal (N), usando-se o símbolo N/ 2 quando a correlação for de $1 \mathrm{mV}=5 \mathrm{~mm}$, ou o símbolo $2 \mathrm{~N}$, quando a $1 \mathrm{mV}$ corresponder um deslocamento de $20 \mathrm{~mm}$ na linha de base; 2) interferências eletromagnéticas; 3) filtro; 4) sistemas de aquisição e processamento do sinal; 5) sistema de impressão.

\section{Bibliografia Consultada}

1. Report of Committee on Electrocardiography, American Heart Association Recommendations for standardization of leads and of specifications for instruments in electrocardiography and vectorcardiography.Circulation $1967 ; 35$ : 583-602.

2. Kadish AH, Buxton AE, Kennedy HL, et al. ACC/AHA Clinical Competence Statement on Electrocardiography and Ambulatory Electrocardiography: a report of the ACC/AHA/ACP-ASIM Task Force on Clinical Competence ACC/AHA Committee to Develop a Clinical Competence Statement on Electrocardiography and Noninvasive Electrocardiology. J Am Coll Cardiol 2001; $38: 7$.

3. Schlant RC, Adolph RJ, DiMarco JP, et al. Guidelines for electrocardiography: a report of the American College of Cardiology/American Heart Association Task Force on Assessment of Diagnostic and Therapeutic Cardiovascular Procedures (Committee on Electrocardiography). Circulation 1985; 85:1221-8.

4. Bailey JJ, Berson AS, Garson A, et al. Recommendations for standardization and specifications in automated electrocardiography: bandwidth and digital signal processing. A report for health professionals by an ad hoc writing group of the Committee on Electrocardiography and Cardiac Electrophysiology of the Council on Clinical Cardiology, American Heart Association. Circulation 1981; 81:730-9.

5. Goldberger JJ, Challapall S, Waligora M, et al. Uncertainty principle of signalaveraged electrocardiography. Circulation 2000; 101:2909-15.

6. Laks MM, Arzbaecher R, Bailey JJ, Geselowitz DB, Berson AS. Recommendations for safe current limits for electrocardiographs: a statement for Healthcare Professionals From the Committee on Electrocardiography, American Heart Association. Circulation 1993; 93: 837-9.

7. AAMI, American National Standard, Safe Current Limits for Electromedical
Apparatus. (ANSI/AAMI ES1-1993). Arlington, Va: Association for the Advancement of Medical Instrumentation; 1993.

8. Pipberger HV, Arzbaecher RC, Berson AS, et al. Recommendations for standardization of leads and of specifications for instruments in electrocardiography and vectorcardiography: report of the Committee on Electrocardiography, American Heart Association. Circulation 1975;52:11-31.

9. AAMI, American National Standard, Safe Current Limits for Electromedical Apparatus. (ANSI/AAMI ES1-1985). Arlington, Va: Association for the Advancement of Medical Instrumentation; 1985.

10. Breithardt G, Cain ME, el-Sherif N, et al. Standards for analysis of ventricular late potentials using high- resolution or signal-averaged electrocardiography: a statement by a Task Force Committee of the European Society of Cardiology, the American Heart Association, and the American College of Cardiology. Circulation $1983 ; 83: 1481-8$.

11. Schlant RC, Adolph RJ, DiMarco JP, et al. Guidelines for electrocardiography: a report of the American College of Cardiology/American Heart Association Task Force on Assessment of Diagnostic and Therapeutic Cardiovascular Procedures (Committee on Electrocardiography). Circulation 1985; 85: 1221-8.

12. Tayler DI, Vincent R. Artefactual ST segment abnormalities due to electrocardiograph design. Br Heart J 1985; 54: 121-128.

13. Gold RG. Do we need a new standard for electrocardiographs? Br Heart J 1985 54: 119-20.

14. Krikler DM, Macfarlane PW. Standards for electrocardiographs. Br Heart J 1974; 36: 945-7.

15. Willems et al. Computer ECG analysis: towards standardisation. Amsterdam: North Holland, 1986:191-3. 